its point to the depth of about 1 millimeter into a metallic
cube full of powdered gas coke, and fixed upon the horizon-

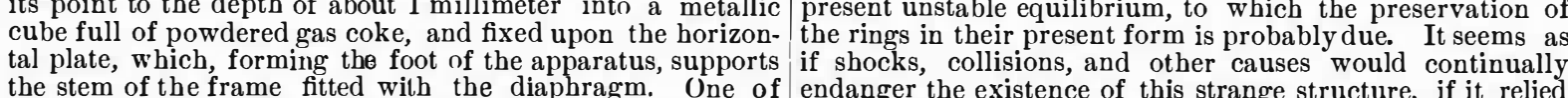
the poles of the battery (a Leclanché of four elements) is endanger the existence of this strange structure, if it relied

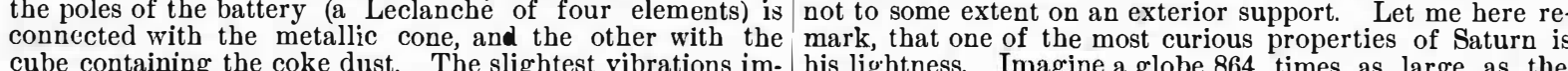
cube containing the coke dust. The slightest vibrations im
parted to the membrane by sound waves suffice to modif the pressure of the cone in the coke dust, thus determining $\overline{\text { THE RINGS OF SATURN }}$

THE most wonderful body belonging to our planetary system is undoubtedly Saturn with its rings and satellites.
During the last two years Saturn has passed through a pe
riod of its journey around the sun, during which it presented itself to us in its most interesting positions. The rings wer repeatedly nearly hidden from view, and, as this takes place
only every fifteen years, the planet was watched with attenonly every fifteen years, the planet was watched with atten-
tion by astronomers at all suitable points. The following, containing a partial description of the dif-
ferent phases as observed at Paris by Camille Flammarion, we take from L'Illustration (No. 1860, October, 1878): The rings of Saturn have now for some time been exposed to our view, and, by means of a telescope of moderate pow-
er, we can now gradually see them disappearagain. When we examine this planet for the first time through and can hardly believe our eyes. Of all the observations I and can hardly believe our eyes. Of all the observations 1 pass from my memory; they are those of the rings of Saturn,
of the moon, and of the triple star (orange, green, and blue) of the moon, and
of Andromeda.

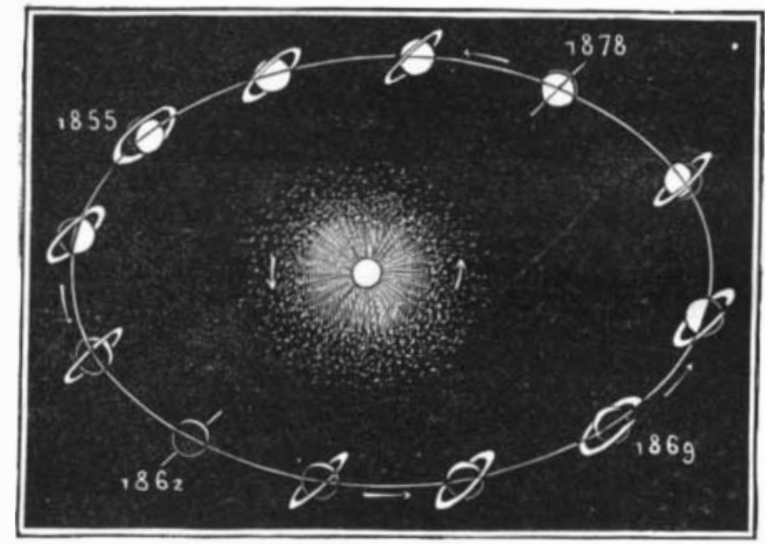

Fir. 1.-CHANGES IN THE ASPECT OF THE RINGS

Truly Saturn presents a unique appearance. The globe
planet proper is at a certain distance surrounded by a or planet proper is at a certain distance surrounded by a
flat round ring. As from our position we only obtain an circular form, but as an eclipse, the small diameter of which is of variable length.

As seen from the earth, one part of the ring seems to
pass through the planet, while the opposite side seems to pass pass through the planet, while the opposite side seems to pass
behind it. Near to where the ring projects itself on the planet its shadow may be observed in form of a dark zone.
The planet is itself not a source of light, but receives it,

The ring does not seem to consist of one single piece, but
The ring is divided into two concentric parts, the separating line being
apparently situated nearer to the outer than the inner edge it is probable, that there are a greater number of rings, as very powerful instruments have shown indications of further subdivisions. One part of the ring is also more luminou
than the other, while the latter is a little more transparent. This is the only instance known of a celestial body with
parlially plane surface, and this is not less than 71.000 miles parliany plane surface, and $11,800 \mathrm{Fr}$. miles in width, but is
only from 60 to 78 kilometers thick. Both sidesare flat and alternately exposed to view, just according to the different positions in which Saturn and earth are placed to each consist of an enormous number of distinct particles, trave ing to their respective distances from the same.

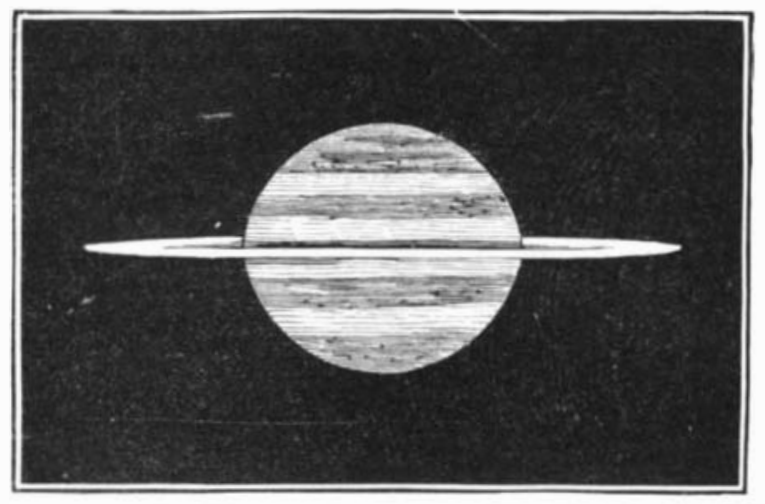

FIG. 2.-ACTUAL VIEW OF SATURN DURING THE SEPTEMBER, 1878.

According to my calculations, the particles forming the planel in from five hours and fifty minutes to seven hours and eleven minutes, according to their distance from the common center of gravity, the zone, being nearest, travel
ing fastest; the particles forming the luminous part of the ring must revolve in from seven hours and eleven minutes to eleven hours and nine minutes. A point on the extreme
outer edge would consume twelve hours and five minutes in accomplishing one revolution around the central globe. The interior edge moves at a velocity of 425 French miles accompanying Saturn at a greater distance may, however, produce deviations from the results as thus calculated. Tu
their influence perturbations may be ascribed, causing the his lightness. Imagine a globe 864 times as large as the
earth, but weighing only 92 times as much! The density earth, but weighing only 92 times as much! The density
of its constituents is seven times lower than the material composing our globe. Saturn would float on an ocean like From time to time the ring disappears from our view in consequence of the compound movement of earth and Sat-
urn, as may be easily explained. Supposing our position to be on a straight line, forming a continuation of the axis of overhead of one of its poles, we would see the ring in its
of rue circular form. Supposing, on the contrary, ourselves would in a plane laid through the equator of Saturn, we truding over its disk on both sides. Between these two extreme positions the ring presents itself in a more or less
oval form, just according as we view it more or less obliqueWe never, however, come near enough to the direction of over balf of the longer one.

Fig. 1 shows the rings as seen by the terrestrial observe
according to the different positions of our globe. It will be een that, at two points diametrically opposed to each other ness of the ring, noticeable only by the most powerful in-
struments, as a fine, white, straight line. This was the case, for the first time since 1862, in June count of the peculiar constellation of both planets, and again shortly afterward on account of being partially
eclipsed. The following table shows the variation of length of the small diameter of the ovals formed by the ring since

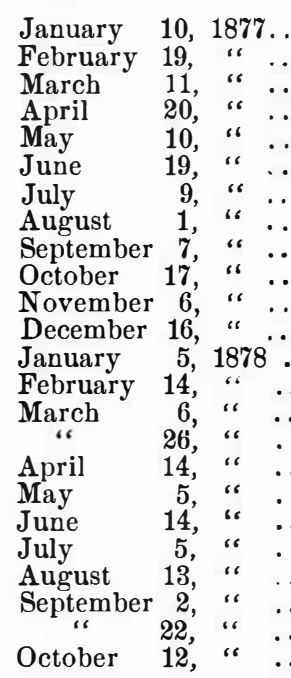

These numbers show how the length of the small diame$\mathrm{r}$ of the perspective view of the rings balances between
certain limits. In June, 187\%, the ring disappeared ; subisible again, reaching, their maximum diameter in Novem-
ber. In March, 1878, we last saw the northern face of the ing, which had been visible since 1862 , and the rings disap-
eared for a second time. At present, the 12th of October, surface of the rings; since then it has steadily approached again the equatorial plane of Saturn, being to-day at an elevation of 229 "above the same. This elevation will con-
tinue to decrease till the rings disappear for a third time. After that we will see their small diameter increase again,
passing from the position indicated on the right, of 1878 passing from the position indicated on the right, of 1878
(Fig. 1), to that on the left, exposing their southern surface to our view till 1885 . In that year the small diameter will to us being symmetrical to that of 1869 . Fig. 2 presents the actual appearance of Saturn during the month of Septem. lar system we just admired. As stated above, it is supplied
with a body guard of satellites, which, as far as our know-
let with a body guard of satellites, which, as far as our know-
ledge, goes, has not its equal within the solar system. Eight verse, representing an empire of millions of miles in area. Hesiod thought he expressed sufficiently the dimensions of the universe when he said that the anvil of Vulcan was
under way nine days and nine minutes to fall from heaven under way nine days and nine minutes to fall from heaven to earth, and just as long to fall from earth to hell. The
distance indicated by this fall, although greater than the lunar orbit, would not equal that of the Saturnian system.
The precise measures of astronomy are incomparably more tion. The system of Saturn, so distant from us that the
head of a pin held at arm's length is sufficiently large to hide it from our view, is not unlike a solar system on a
small scale; we must say comparatively on a small scale, as small scale ; we must say comparatively on a small scale, as
the minute disk of the smallest satellite, as seen by us with the most powerful telescope, represents an area of million small in creation, and, worthy of our attention as this sys-
tem is, it constitutes for its inhabitants really a universe for itself.

What are, finally, the conditions under which this republic celestial bodies could be inhabitable? What similarities In what form could organic life exist on that star and its

The inhabitants of such a body must certainly differ widely from terrestrial beings. The light specific gravity of
Saturn and the density of the atmosphere would necessarily imply a development of vital organization in unimagina-
ble forms. To suppose, as it is probable, that there be nothing solid, that the planet itself were not a solid but a liquid
ing ody, that the living beings were of a soft, gelatinous mateNevertheless, there are more reasons speaking in favor of Nevertheless, there are more reasons speaking in favor of
such a theory regarding Saturn than in reference to any
other body belonging to our solar system. Its conditions of only the edge of the ring is visible, and owing to the thin equently the earth rose to some distance above the equatoe see the southern face to a limited extent yet; on the 24 th ber last.
The ambition of Saturn was not contented with the annu-
. poetical than the fictions of poets of the boldest imagina- gravity are not only peculiar in general, but they vary even If we could penetrate into all of Nature's mysteries, we might perhaps find that the Saturnians have transparen bodies, through which the circulation of life might be ob-
served; that they never feel the weight of matter; that they y without wings in midst of a nutritious atmosphere; that tion and its ridiculous consequences; that they are gifted with a nervous system incomparably more sensitive than Whies of creation, and live in an angelic state a life thirty
eries No matter to what extent and by what forms of life Saturn may be inhabited, we need not doubt that nature has ner to, as it has done it with, our moderate terrestrial facili-

[The Australian Medical JournaL.]

PEROXIDE OF HYDROGEN IN THE BLOOD.

ATtempt to Explain the curative action of By John Day, M.D.*

Although medicine has not yet attained the rank of an exact science, we are, fortunately, often enabled to call to our aid chemistry and other exact sciences for the purpose nature and treatment of disease; and I shall endeavor thi o show you that an approach at least, toward a scientific explanation, may be offered for the use of a rather large number of our most favorite remedies. I have selected for viz: : Cod-liver oil and other fixed oils and fats, oils of turpentine and other essential oils, common resin, the resin of ray of medicines in general use, and it will be my aim to show
you that, al though most of them differ widely in their general characteristics, they all possess one property in common, viz., the power of absorbing atmospheric oxygen and conorting it into peroxide of hydrogen-a substance possessed
of many curious and interesting properties, some of which or many curious and interesting properties, some of which
are at present but little understood. It is composed of $t w o$ atoms of hydrogen and two atoms of oxygen, hence its symbol is $\mathrm{H}_{2} \mathrm{O}_{2}$, and its combining weight 34 . This com pound is remarkable for the ease with which it parts with its second atom of oxy gen, which is held in very loose com-
bination; and also for the readiness with which, under cerbination; and also for the readiness with which, under certhis decomposition may be effected in a variety of ways, but gen is brought into contact with the coloring matter of blood, with tibrin, or with pus, is a property which renders
this substance highly interesting in a physiological point of

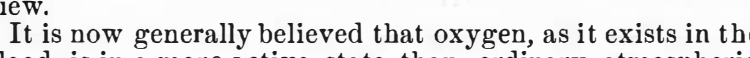
oxygen. Carpenter says: "As regards the oxygen contained

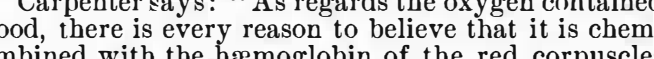
in which it exists in the active or ozonized condition."

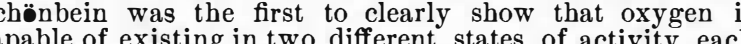
capable of existing in two different states of activity, each ordinary oxygen was a perfectly neutral gas, and incapable producing any oxidations until it had been chemically idered that the oxygen absorbed during respiration was thus acted on by the coloring matter of the blood; and the views
am about to submit for your consideration are to some ex ant a modification of those entertained by him. Instead, owever, of believing, as Schönbein did, that the first change coloring matter of the blood, I hold that the first change is produced by the fat, which exists in the blood in considerble quantities, more particularly in the corpuscles; and I assume that this fat, which is in a state of extensively fine
subdivision, is incessantly engaged in acting on the inspired axygen contained in the red corpuscles, and converting it into peroxide of hydrogen-a substance which is instantly
transformed by the coloring matter of the blood into water and oxygen-a gas which at the noment of its liberation is possessed of many of the characteristics of ozone, and is believe that it is oxygen in this state, although perhaps no always liberated from peroxide of hydrogen, which gives mal body during life.

INTERESTING CHEMICAL EXPERIMENTS.

I will endeavor to demonstrate the correctness of this

theory by a chemical experiment. Oxygen in a nascent state, like ozone, is capable of oxidizing and turning blue
the resin of guaiacum, but neither the oxygen contained in proxide of hydrogen nor atmospheric oxygen is capable which, like all animal fats that have been deprived of blood nd freely exposed to the air, contains spontaneoustly genrated peroxide of hydrogen. I will first place a little of it
on a piece of white blotting paper, and then pour over it not, you will find, be followed by any change of color in the ot, you will find, be followed by any change of color in the uaiacum, the oxygen of peroxide of hydrogen being incapa-
ble of oxidizing and turning it blue; but, on the addition of
small quantity of the coloring matter of blood, you will small quantity of the coloring matter of blood, you will perceive that a bright blue reaction will quickly ensue,
howing that oxidation of the guaiacum has occurred.

\section{PEROXIDE OF HYDROGEN.}

This result has been brought about by the coloring matter f the blood having transformed the peroxide of hydrogen contained in the fat into water and nascent oxygen. To coloring matter of blood alone is incapable of oxidizing and rning blue the resin of guaiacum

In stating to you just now that all animal fats which have been deprived of blood and exposed to the air contain pertaining blood were incapable of generating peroxide of hy drogen, but simply that, so long as any of the coloring matter of blood remained within them, the peroxide of hy
would be decomposed as rapidly as it was generated. 
\begin{tabular}{l|l|l} 
I cannot, of course, show you that fat, as it exists in the & it is liberated by blood. The purifying and stimulating ac- \\
living body, is capable of converting the inspired oxygen into & tion of common resin, when applied to bleeding or sup-
\end{tabular} living body, is capable of converting the inspired oxygen into peroxide of hydrogen; nor can I show you that, during life,
the fibrin and the coloring matter of the blood are capable of the fibrin and the coloring matter of the blood are capable of Guaiacum resin, once a famous remedy for chronic
d composing peroxide of hydrogen and resolving it into 'rheumatism, is still recommended by Dr. Garrod and some
water and oxygen; bu I can clearly show you that animal : other practitioners of eminence in the treatment of this dis fats, out of the body, possess the property of generating ease. It is, however, rather uncertain in its action, and this pcroxide of hydrogen, a nd of concentrating and storing it up, substances which are capable of reducing it and liberating its oxygen.
I have alreatly shown you that spontaneously generated
peroxicle of hydrogen exists in human fats, and I will now, peroxide of hyd roreme exists in human fats, and I will now,
by means of the same test, which is perfectly reliable, offer you proof of its existence in the fats of mutton and beef, capable of oxidizing guaiacum resin in the presence of blood. I will then proceed to show that the same principle exists in
almond, olive, and linseed oils; in the essential oils of turpentine, juniper, lavender, peppermint, and caraway,

common resin, the resin of guaiacum, myrrh, and ether.
It has been stated by Professor Roscoe and other modern
writers on chemistry that peroxide of It has been stated by Professor Roscoe and other modern
writers on chemistry that peroxide of hydrogen does not oc-
cur in nature, and I may bere venture to state that I claim cur in nature, and I may bere venture to state that I claim
to have been the first to show the presence of this physiologically interesting principle, not only in fats and oils, but also in a very large number of substances, many of which rank
among our most valuable remedial and prophylactic agents. among our most valuable remedial and prophylactic agents.
The importance of fat in the animal economy is now more clearly recognized than it formerly was. Dr. Sydney Ringer,
in his well-known work on Therapeutics, says: "Fats are necessary foods to the animal body, being both heat-giving, force-supplying, and plastic. Their combustion
mainly to the generation of the heat of the body.

Having demonstrated to you the fact that, apart from the
conditions of life, all fats and fixed oils possess the property conditions of life, all fats and fixed oils possess the property
of absorhing atmospheric oxygen and converting it into of absorhing atmospheric oxygen and converting it into
peroxide of hydrogen-a substance which, as I have said
before is resolved into water and nascent oxygen when before, is resolved into water and nascent oxygen when
brought into contact with the coloring matter of blood, I will now assume that fats in the living lorly obey the same
chemical laws as they do out of the therly, and that the oxygen which has been absorbed by the red corpuscles of the blood during respiration is converted by the fat which is al-
ways present in the blood, although in varying proportions, into peroxide of hystrogert, and that the peroxide of hydrogen
thus formed is resolvet by the coloring matter of the blood into water and nascent oxygen.

$$
\text { EFFECTS OF IRON IN THE BLOOD. }
$$

I think it possible that the iron in the coloring matter of the blood may be the principle which decomposes the per-
oxide of hydrogen and liberates its oxygen, and I will show you an explerisrnent. which rather favors this view. The exof our first experiment, in which I showed you that the
oxygen of peroxide of hydrogen, as contained in the human fat, could not. by itself, oxidize the resin of guaiacum; but
that on the addition of a mere trace of a watery solution of the coloring matter of blood it did so reatdily. On this oc-
casion the only difference I shall make will be to substitute a watery solution of perfectly pure protosulphate of iron for the solution of the coloring matter of the blood, and we
shall find that the guaiacum resin will be oxidized and turned blue, just as it was by the coloring matter of blood.
Protosulphate of iron which has become, even in the slightest degree, oxidized, is unfit for this experiment, as it would
then contain ozonic oxygen, and would oxidize guaiacum resin and turn it blue without the intervention of peroxide of hydrogens
Wher this peculiar, but at present barely recognized
property of fats and fixed oils, becomes more generally (and in the animal pronomy regarding the functions of fat in the animal economy; and also, perhaps, to a clearer knowledge of the therapeutic in-
fluence of fats and oils. Cod-liver oil, by common consent,
takes the highest place as a remedial agent among the fats. The ease with which it is digested may, perhaps, be its
chief recommendation, for we often find that those who have derived the greatest benefit from its use have been un-
able to digest other fats. Its therapeutic value is universally recognized in the treatment of phthisis, scrofula, and other
diseases in which the functions of oxidation and nutrition are imperfectly performed; and I am disposed to attributio some
of its curative properties to the power it possesses of readily carrying peroxide of hydrogen into the circulation, and of of strumous and tubercular matter.

SUBSTANCES WHICH GENERATE PEROXIDE OF HXDROGEN IN

To this property of spontaneously generating peroxide of
loydrogen, coupled with a power of readily entering the lyydrogrn, coupled with a power of readily entering the
ljlood when administered internally, may, I think, be
ascribed the beneficial action of miny other therapeutic agents, some of which experience has shown to be of value
in the treatment of diabetes, gravel, rheumatism, and gout, all of which rank among the diseases of sub-oxidation.
Many years ago the use of what was called ozonized codliver oil was hitiphly recommended by Dr. Symes Thompson
and his father. In twenty cases in which it was adminis-
tered by the latter gentleman the pulse was reduced more tered by the latter gentleman the pulse was reduced more in seven of the remainder. Now, this so-called ozonized oil ly charged with peroxide of hydrogen in consequence of long exposure to the direct rays of the sun. I may ob-
serve that the eombined influence of warmth and light greatly expedites the formation of peroxide of hydrogen.
During the last few years Dr. Balthazar Foster, of Bir mingham, has strongly advocated the use of etherized cod-
liver oil in the treatment of phthisis. Now, here again we have oil in the treatment of phthisis. Now, here again w
of hydrogen than containing a larger proportion of peroxid
indinarily contains, and this is due to of hydrogen than it ordinarily contains, and this is due to
its admixture with ether, a substance which possesses a
much bigher power of generating peroxide of hydrogen much higher power of generating peroxide of hydrogen of hydrocarbons and oxyhydrocarbons, possesses the property
of absorbing atmospheric oxygen and converting it into peroxide of hydrogen; and this property, coupled with the decomposition and liberation of nascent oxygen, which takes
place when peroxide of $h_{2 y d r}$ rerer is brought into contact with place when peroxide of hydrefrer is brought into contact with
either blood or pus, atfords, I think, a tolerably rational explanation of the beneficial action of tincture of myrrh in the
treatment of bleeding and spongy gums, and of certain forms of sore throat. It is and that of sore throat. It is worthy of remark that pus liberates the is, that this douching is entirely painless after a few appli-
oxygen from peroxide of hydrogen much more rapidly than cations. It may smart a little the first or second time, but even then it is so slight as not to be taken into considera tion. Now to mention a few cases, as it would only be te-
dious to give them all. The following are fair samples of the whole, as they are taken entirely at random and not semarked example of the efficacy of this treatment: CAsE.-C. K., male, aged 29, was sent to the hospital to
have the leg amputated at the middle third of the thigh, as have the leg amputated at the middle third of the thigh, as
a last resort. Had an ulcer three inches by one and a half an last resort. Had an ulcer three inches by one and a dateral aspect of the leg. The limb was
swollen to twice the natural size, and gangrenous in appear swollen to twice the natural size, and gangrenous in appear
ance as far as the lower third of the thigh. The discharge from the ulcer was thin, sanious, and very fetid. There was no bistory of syphilis, either congenital or acquired. Had had tried almost everything, and seen a number of physicians in regard to it. He walked with great pain and diff-
culty. The solution of chlorate of potassium was used, and by the third day a decided improvement was noticed. The
swelling of the leg had decreased, and the ulcer began to granulate nicely. At this time he had an attack of delirium tremens, which lasted four or five days, but notwithstanding this shock to the nervous system, the leg continued to
improve visibly from day to day. At the end of four weeks the limb was of the normal size and appearance, the ulcer healthy, and about as large as a three cent piece. The pa-
tient could walk without any inconvenience, and insisted on tient could walk without any inconvenience, and insisted on
leaving the hospital to fill the position of organist in a Catholic church, although strongly ad vised not to. He returned after a week or two with the leg slightly swollen, and the
ulcer about the size of a silver quarter. The treatment was resumed, and he was discharged cured in ten tlays.
CAsE.-A. G., female, aged 30. Syphilitic. Larme deep CAsE.- - A. G., female, aged 30. Syphilitic. Lurrese deep
ulcer on the posterior aspect of leg. Discharge excessively foul and unhealthy. Had tried specific treatment alone in
vain. Leg healed ncely in about five weeks under the com-
bined use of constitutional and the chlorate of potassium CASE.-M. W., female, aged 65 . Syphilitic. An ex-
tremely large and deep ulcer, covering nearly the whole of tremely large and deep ulcer, covering nearly the whole of
the posterior and external lateral aspects of the leg. Discharge excessively fetid. Rapid and continued improvement for several weeks under the combined constitutional an
chlorate of potassium treatment. Lost sight of before com plete healing took place.
chlorate of potasium

CASE.--J. H., male, aged 66. Several small but trouble-
some ulcers along the crest of the tibia. Had been troubled some ulcers along the crest of the tibia. Had been troubled
for months. Probably due to a previous injury of the kneefor months. Probably due to a previous injury of the knee-
joint. Healed completely in ten days by this treatment. CASE. - P. M., male, aged 38. Varicose ulcer of leg. Had week.
CASE.-O. H., male, aged 46. Small indolent ulcer of the CAsE.-O. H., male, aged 46. Small indolent ulcer of the
leg of matry months' standing. Healed in one week.
. CAsis - C. K., female, aged 40. Large indolent ulcer of
the leg of over a year's standing. Rapid and continued im provement for two weeks. Was then lost sight of.
CASE.-P. N., male, aged 38. Troubled with a

icose ulcer for several years. Healed completely

HE TREATMENT OF ULCERS WITH A SATU By T. M. Rochester, M.D.

I wish to call attention to the treatment of ulcers, ulceraions, and suppurating wounds and sores in general, by the said suppurating sores in general, and so it will be found
beneficia in all of these, but it is especially useful in that particular class of old, unhealthy, indolent ulcers which are
one of the opprobria of our art. Let me describe briefly my plan of treatmentin one of these cases.
Suppose, for example, a patient comes with an old, indoThe sore on the legl, unhealthy in appearance, and ill-smelling.
The limb is usually much swollen, granulation is at a standThe limb is usually much swollen, granulation is at a stand
still, the ulcer may even be phagedenic, and the discharge is
thin, sanious, and offensive. As a rule, the patient has the thin, sanious, and offensive. As a rule, the patient has the
sore covered with some salve or ointment, and the limb

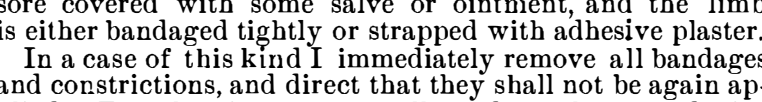
plied. The ulcer is then carefully and gently washed with
warm water after which it is ordered to be gently douched warm water, after which it is ordered to be gently douched
five or six times a day with a saturated solution of chlorate
of potassium. My usual prescription is: Take of pot. chloratis, 2 drachms; aquæ puræ, one pint. The best method of doing this is to fill a sponge with the solution, and then
slowly press it out, holding it within an inch or two of the

After the ulcer has been quite freely douched, a thin layer of the unguentum zinci oxidi should be spread around the oiled lint, kept in place by a bandage applied as loosely as
possible. The douching should be repeated at first five or possible. The douching should be repeated at first five or
six times daily; the zinc ointment need not be used mor bree days the ulcer wijl begin to assume a healthy appear
ance, and commence to granulate nicely. When this im provement is noticed, the number of douchings should be
decreased otherwise the granulations will become pale and decreased, otherwise the granulations will become pale and
flabby. As the sore fills up and begins to heal over around flabby. As the sore fills up and begins to heal over around Sover the new skin. This serves to protect and strengthe
So, too, it is well to continue its use for a few days atter Such, in brief, is my mode of dealing with these cases
and it is with the keenest satisfaction that I have watched and it is with the keenest satisfaction that I have watched cerations, which have defied one treatment after another, and worn out the patience of physician and patient alike, treatment, and thence proceed rapidly to a complete re-
covery. During the past fifteen months I have seen and
treated by this plan bet ween nintrty and one hundred cases, nd in no single instance have failel to see immediate and ontinued improvement, and, when they could be kept track
f complete healing in from one to six weeks. This number of cases embraces not only the ordinary in which I believe was carcinoma tous in its character.
Several of my cases had scritpiny and skin grafting tried vain. Where they were s.pphilitic, constitutional treat ent had been and was used alone without effect, but rapid ealing immediately took place when the douching with The saturated

There is another point that is worth mentioning, and that
THE Chemiker Zeitung reports that the Wesenberg Chiccory
a, that this douching is entirely painless after a few appli-
Works of Berlin were hurnt to the ground on the 4th Sept.
Weeks. $-S$. T., female, aged 10. Simple but unhealthy-
looking ulcer of several weeks' duration. Healed in one CAsE.-M. C., female, aged 40 . Varicose ulccr of several years' tandling., Healed in four weeks.
CAsE. - F. F., male, aged 19 . Two large, ragged, deep and unhealthy-looking sores on the right leg. and a similar though gangrene had set in. Healed completely in three
weeks. weeks.
CASE.-M. M., female, aged 40. Iarge indolent ulcer of the leg of several years' standing. Healed in five weeks.
CASE. - L. C., male, aged 41. Troubled for years with an indolent ulcer of the leg. Had tried almost everythingHealed in two weeks.

And so I might go on and enumerate case after case, but I think the above are sufficient to prove the efficacy of this
plan of treatment. I wish, however, to quote one case, as plan of treatment. I wish, however, to quote one case, a
showing the advantage in the use of the saturated solution ration is profuse.
CASE.-H. B., male, aged 28, of fine muscular develop ment. Recived a penetrating lacerated wound of the arm,
which severed the brachial artery. The hemorrhage was which severed the brachial artery. The hemorrhage wa controlled by acupressure both above and below the wound.
On the removal of the needles a large slough came away, The solution of chlorate of potassium was used, the exces. One word as regards the mode of operation of chlorate of
Ond of potash thus used. I do not know that I am right, but my
theory is as follows: 1st. Its astringent properties cause it to repress exuberan

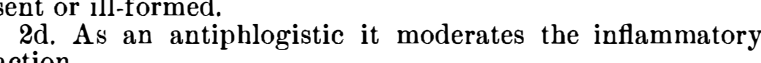
3d. And this I regard as the most important: as an antiseptic it destroys and prevents the growth of bacteria.
This threefold action I believe meets all the indications for the local treatment of this troublesome class of afflic-
tions. In conclusion, let me say that this use of chlorate of potassium is not m y own discovery, but was suggested to me while interne at the Monroe Co. Hospital by the attend-
ing physician, Dr. Azel Backus, of Rochester, who has used

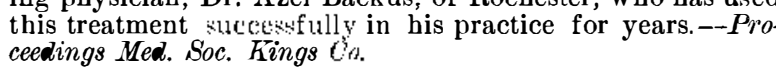

New Method of Detecting Arsenic. - The substance poured into a mixall beaker, and a piece of or sodium amalgam then covered as rapidly as possible with a piece of white filter paper or a porcelain lid, previously moistened with a
weak feebly acid solution of silver nitrate. The presence of arsenic is indicated by a blackening of the paper or of the traces-from strongly alkaline liquids, the solution should
be rendered distinntly alkaline. In this manner the confu-
sion of antimony atrut arsenic is rendered improbaible, though not quite excluded. Organic matter does not interfere.Archiv Pharm., American Journal of Pharmacy. ranulations, or t orcelain. As antimony hydride is not given off-or in mere 